\title{
AC 2008-722: ENGINEERING MATHEMATICS FOR MECHANICAL ENGINEERING STUDENTS: BRIDGING THE KNOWLEDGE GAP
}

\section{Kendrick Aung, Lamar University}

Kendrick Aung is an associate professor in the Department of Mechanical Engineering at Lamar

University, Beaumont, Texas. He received his Ph.D. degree in Aerospace Engineering from University of Michigan in 1996. He has published over 60 papers in international journals and conference proceedings.

\section{Jiang Zhou, Lamar University}

JIANG ZHOU is currently an assistant professor with the Department of Mechanical Engineering, Lamar University, Beaumont, Texas. She received her Ph. D. in Mechanical Engineering from the University of Maryland at Baltimore County (UMBC), Baltimore, Maryland, in May 2003. Her research interests include mechanical applications in microelectronics, biomechanics, system dynamics, and system optimizations, etc.

\section{Chang Li, Lamar University}

X. CHANG LI is currently an assistant professor with the Department of Mechanical Engineering, Lamar University, Beaumont, Texas. He received his Ph. D. degree in Mechanical Engineering from Clemson University. His research interests include gas turbine cooling technologies and energy conservation. 


\title{
Engineering Mathematics for Mechanical Engineering Students: Bridging the Knowledge Gap
}

\begin{abstract}
When teaching mechanical engineering courses to the undergraduate students, mathematics plays a major role in developing mathematical models, implementing solution algorithms for the models, and arriving at the solution. As a result, all mechanical engineering curricula require that students complete a significant number of mathematical courses before they start taking the engineering science and engineering courses. However, many students have difficulty relating and applying mathematical knowledge to the engineering principles and problems that they need to master for their undergraduate degree. There seems to be a huge knowledge gap or disconnect between mathematics and engineering that needs to be addressed so that the students become proficient in applying and integrating mathematics in their engineering studies. This paper provides many examples as well as some solutions to these knowledge gaps between mathematics and engineering from the viewpoint of a faculty member teaching mechanical engineering courses. These examples and solutions may prove to be beneficial to faculty members encountering the same or similar problems in teaching and conducting engineering courses.
\end{abstract}

\section{Introduction}

It is common knowledge that engineers must be proficient in many topics of mathematics such as linear algebra, numerical methods, and differential equations in order to tackle and solve realworld engineering problems in their daily work. As a result, education of engineers involves completion of a number of mathematical courses early in their engineering curricula. For example, at Lamar University, mechanical engineering curricula requires that students complete 15 credit-hours of mathematics before their junior year. The courses include 3 classes of Calculus and Analytic Geometry, 1 class of Linear Algebra and 1 class of Differential Equations. In addition, the students need to take one statistics course in their senior year before their graduation. All the mathematics courses except the course on statistics are taught by faculty from the Department of Mathematics. The statistics course is taught by a faculty from the Department of Industrial Engineering.

The importance of mathematics in engineering curricula is so important that ABET includes proficiency in mathematics as a specific program outcome in their accreditation process ${ }^{1}$ : Outcome (a) an ability to apply knowledge of mathematics, science, and engineering. However, for the Mechanical Engineering program at Lamar University, two more program outcomes ${ }^{2}$ on mathematics in addition to ABET outcome (a) are included: Outcome (1) an ability to apply advanced mathematics through multivariate calculus and differential equations and Outcome $(\mathrm{m})$ an ability to use statistics and linear algebra. These two outcomes are included due to recommendation from the relevant professional society, in this case, American Society of Mechanical Engineers (ASME). 
After the sophomore year, mechanical engineering students start taking engineering courses relevant to mechanical engineering such as fluid mechanics, system dynamics, and heat transfer. Even though all the junior students have completed and received passing grade in these mathematic courses, a majority of them have found it difficult to relate and apply the mathematical knowledge that they have acquired in their engineering classes. There seems to be a huge knowledge gap or disconnect between mathematics and engineering that needs to be addressed so that the students become proficient in applying and integrating mathematics in their engineering studies. This paper provides many examples as well as some solutions to these knowledge gaps between mathematics and engineering from the viewpoint of a faculty member teaching mechanical engineering courses.

\section{Examples of Knowledge Gaps}

An example will clearly demonstrate the issue of knowledge gap typically encountered in mechanical engineering courses. In an engineering thermodynamics class, the change in property of a substance such as enthalpy needs to be determined. Since enthalpy is a property, the change in its value depends only on the two end states, independent of the path between the two states. The mathematical topic underlying this example is exact differential. According to Moran and Shapiro $^{3}$, the differential of every property is exact. Every engineering student has studied the topic of exact differential in their mathematic class but many failed to realize the application of exact differential in their thermodynamic class. More examples will be given as found in the typical mechanical engineering courses.

\section{Engineering Static and Dynamics}

Engineering mechanic courses are the first engineering courses that students encounter in their sophomore year. One of the early uses of analytical geometry in this class is to determine the center of mass or centroid of different physical bodies. The students can determine these computations for simple shapes but many students have difficulty in choosing correct differential element, applying the correct limits, and performing the integral for complex bodies which are very common in engineering mechanics. These difficulties are more evident when the students are asked to determine the area or mass moment of inertia of complex bodies. One of the reasons may be due to lack of ability to visualize the 3-dimensional bodies on the part of the students.

Another area where students could not relate between mathematics and engineering is graphical relation between the process of integration and differentiation. In dynamics, parameters such as displacement, velocity, and acceleration of a particle motion can sometimes be represented in graphical form. For example, from a given graph of an acceleration of a particle, the displacement of the particle during a certain time interval needs to be determined. In this case, many students discover that they cannot perform graphical integration correctly even though they can do the operation if an equation for the acceleration is given. In both examples, the real problem for students is that they cannot apply the mathematical concepts and solution methodology they have learned in their analytic geometry class to the engineering problems. For example, they can perform integration, $\int x d x$, to obtain the solution, $x^{2} / 2$ but fail to see the integration process graphically, i.e. integrating a strain line will result in a parabola. 


\section{Fluid Mechanics}

Mechanical engineering students take MEEN 3311 Fluid Mechanics during their junior year at Lamar University. In this course, the major difficulty encountered by students is on the topic of multivariate calculus or differential equations. In fluid mechanics, conservation equations for mass, momentum, and energy are derived mathematically, and solved for some simple flows. In the text used at Lamar University, the topic is covered in the chapter called the differential analysis. In this chapter, simple flows such as flow between parallel plates are solved analytically from the governing differential equation. As discussed earlier in the paper, differential equation is a required math course completed by the students in their sophomore year. However, almost all students have difficulty in solving the Navier-Stokes equations together with the conservation of mass equation. The solution methods involved are quite simple but students have difficulty in transforming, for example, the physical boundary to a mathematical equation, or understanding the analytical solution in relation to the physical problem. This difficulty is not limited to this course, but in many courses as well. In this case, the major problem is inability to relate physical and mathematical knowledge, since many students see differential equations as equations to solve rather than as representation of a practical flow such as flow of water in a round pipe. The consequence of this shortcoming of students is that they also have difficulty interpreting and understanding what the analytical solution represents in the real-world problem.

\section{Heat Transfer}

Similar to Fluid Mechanics, the subject of heat transfer is heavily reliant on mastery of mathematics, especially differential equations. The solutions for heat conduction problems are generally obtained through solution of the differential equation for the given problem. The difficulties encountered are similar to the subject of fluid mechanics: applying proper boundary conditions and solution methods, and interpretation and visualization of solution obtained from the differential equation. For example, in an unsteady heat conduction problem, the solution will tend to equilibrium or zero when the time is long, and as a result, the solution typically includes an exponential decaying term in time. However, if you ask the students to sketch the solution (without using the analytical exact solution) for an infinite time, they do not realize that the solution decays exponentially. Therefore, understanding of different mathematical functions and their characteristics must be emphasized in class to address this difficulty.

A new problem area encountered in the subject of heat transfer is the application of numerical solution methods to the governing differential equations. In many textbooks on heat transfer, numerical solutions of heat transfer problems are included. In solving numerically, finite difference method is typically used, and the students are required to solve a set of algebraic equations numerically. Generally, the text book covers both direct methods such as Gauss Elimination as well as indirect methods such as Gauss-Seidel iterative method. As discussed earlier, the students are required to complete a linear algebra course where solutions of algebraic equations are covered. However, many students lack understanding of the nature of algebraic equations they solve and try to use mathematical software packages such as Matlab or their calculators to solve these equations. For example, many students try to solve the equations given below and obtain results that satisfy the equations using either Matlab, MathCAD, or a program in their calculator. 


$$
\begin{gathered}
3 x+4 y+6 z=20 \\
6 x+8 y+12 z=40 \\
x+3 y+7 z=12
\end{gathered}
$$

If one looks at the equations carefully, it is easy to realize that first and second equations are identical and therefore there is no unique solution to this set of equations. However, the mathematical software packages will still solve and produce the solutions which obviously are different from one solution to another. The lesson here is that the students must understand issues such as convergence and numerical errors when dealing with numerical solutions of engineering problems. The students also need to understand that the numerical solutions are approximate solutions, and they must be able to justify the convergence and validity of the numerical solution.

\section{System Dynamics}

A course in system dynamics that deals with mathematical modeling and response analysis of dynamic systems is required in most mechanical and many other engineering curricula. The analysis and design methods in the course cover a wide variety of different systems, such as mechanical, electrical, pneumatic, hydraulic, and thermal systems. Although systems are in various fields, mathematically they all can be simplified and represented by ordinary differential equations. Mastering ordinary differential equations (ODEs) is therefore very important and essential to being successful in this course.

Basic concepts of solutions of first and second order differential equations and Laplace transforms are expected to be firmly planted in the students' skill sets. However, the reality is that the students simply do not remember much of former material since these courses were taken a year, or even years earlier. Moreover, new terminology and concepts, such as transfer function, poles, zeros, stability, and others cause even more confusion for the students. In teaching the Dynamic Systems course at Lamar University, the instructor uses a number of lecture periods to review the topics of Laplace Transform and solutions of ordinary differential equations to facilitate students' learning.

\section{Some Suggested Solutions}

The examples given above are not necessarily the only problems related to mathematics in terms of undergraduate students. The authors teach graduate courses and similar problems can be common even in graduate classes. Some of the problems may be related to more and more dependence on the computer tools and software packages to solve engineering problems even in the undergraduate courses. Some of the suggested solutions are given below:

(a) At the beginning of the semester, review the mathematical topics relevant to the class, and provide materials as well as conduct a short quiz on the topics. In this way, the instructor will be well aware of the areas of weaknesses among the students and provide additional emphasis as needed during the semester. 
(b) Emphasize graphical representation of mathematical functions and solutions. Provide both analytical and graphical solutions for comparison purposes. Ask students to include graphs of the analytical solutions wherever possible.

(c) Explain the physical meanings of various mathematical functions and individual terms of differential and integral equations. Provide mathematical translation of physical parameters and boundaries in defining these equations.

(d) Integrate analytical and numerical solution methods to tackle the same project. Analytical solutions are required for the better understanding the engineering topics and numerical approaches may help to visualize the physics and results behind the equations. By showing the application of both analytical and numerical techniques to the same problem, students are inspired to learn the resulting similarities and differences.

(e) In numerical solution methods, make students aware of the limitations of solution algorithms as well as numerical errors associated with different numerical methods. Discuss issues of error propagation, convergence conditions, unique solutions, etc.

Some of these solutions are presently being implemented in the current semester in courses such as Systems Dynamics and Heat Transfer. The results of these implementations and analysis on their effectiveness will be available after the current semester is over in May 2008. Based on the suggestions of the reviewers, a general survey of students and mathematics faculty will be done in this semester.

\section{Conclusions}

In this paper, some of the common problems encountered by undergraduate students in different mechanical engineering course regarding applications of mathematics to engineering subjects are presented. Some suggested solutions to these problems are also provided. These solutions are being implemented in several courses, and full results will be made available after the present semester is over. These examples and solutions may prove to be beneficial to faculty members encountering the same or similar problems in teaching and conducting engineering courses.

\section{Bibliography}

1. 2008-2009 criteria for accrediting engineering programs, ABET, 2007. www.abet.org/2011-30-07.pdf

2. http://dept.lamar.edu/mechanical/meweb/proOut.htm

3. Moran, M. J., and Shapiro, H. N., "Fundamentals of Engineering Thermodynamics," John Wiley \& Sons, 1988. 